\title{
Ion Correlations due to a High-Frequency Electric Field and Their Effect on the Nonlinear Plasma Conductivity
}

\author{
JUAN R. SANMTARTIN* \\ Plasma Physics Laboratory, Princeton University, Princeton, New Jersey \\ (Received 7 March 1969; final manuscript received 10 December 1969)
}

\begin{abstract}
The influence of a strong, high-frequency electric field on the ion-ion correlations in a fully ionized plasma is investigated in the limit of infinite ion mass, starting with the Bogoliubov-BornGreen-Kirkwood-Yvon hierarchy of equations; a significant departure from the thermal correlations is found. It is shown that the above effect may substantially modify earlier results on the nonlinear high-frequency plasma conductivity.
\end{abstract}

\section{INTRODUCTION}

The present investigation is primarily devoted to determine whether a high-frequency electric field $E$ affects the ion-ion correlations of a fully ionized plasma in the limit of infinite ion mass $m_{2}$; we show that an effect does exist and obtain an expression for the correlations spectrum that only for vanishingly small $E$ reduces to the known thermal value. The influence of this effect on the nonlinear highfrequency plasma conductivity is studied later; we show that earlier results on the conductivity may be substantially modified by including the above effect.

At first sight, it might seem surprising to find an influence of the field on the ion correlations for $m_{2} \rightarrow \infty$. To clarify our result we notice the following: We consider times large compared with the ion correlation relaxation time, $\bar{\gamma}_{2}^{-1}\left(\bar{\gamma}_{2}\right.$ being some average of the ion Landau damping; as $m_{2} \rightarrow \infty, \bar{\gamma}_{1} \rightarrow 0$ ). Thus, we assume that the field was switched on at time $-\tau$ very far in the remote past, that is, we let $\tau \bar{\gamma}_{\imath} \rightarrow \infty$ so that some oscillating equilibrium is reached; then, we let $m_{2} \rightarrow \infty\left(\bar{\gamma}_{\imath} \rightarrow 0\right)$. Our analysis then shows that the resulting ion correlations are nonthermal. An explanation for this may be given:

The correlations result from an energy balance and all the electron energies have to be included in this balance ${ }^{1}$; it is the energy of the field-induced oscillations of the electrons that produces the departure from the thermal value. In other words, the Debye cloud of electrons around each ion is distorted by the additional energy, with the consequence that the distance between two clouds (that is, between two ions) is changed.

The above result is important because it affects the response of the plasma to the field, and, in particular, the value of the electric conductivity. Since the effect is essentially nonlinear (vanishing as $E^{2}$ for small $E$ ), it must be considered only if nonlinear effects are important. Therefore, we include a discussion of the influence of the above result on the nonlinear high-frequency plasma conductivity.

Nonlinear effects on the high-frequency plasma conductivity have been investigated by Silin, ${ }^{2}$ who used the BBGKY hierarchy of equations but failed to include collective phenomena, and by Albini and $\operatorname{Rand}^{3}$ who assumed a driving frequency $\omega$, large compared with the electron plasma frequency $\omega_{p}$; thus, all typical resonant effects ${ }^{4}$ were missed in both papers. Such effects were properly considered by Kaw and Salat, ${ }^{5}$ who used a physical model first introduced in the study of the linear conductivity. ${ }^{4}$ However, uncorrelated ions were assumed; although the use of thermal correlations in computing the conductivity would yield no essential corrections (see the discussion in Ref. 4 for the linear case), the nonthermal correlations found here do yield essential modifications that invalidate some results of Ref. 5.

In Sec. II we obtain the ion-ion correlation spectrum by solving the coupled BBGKY equations for the ion-ion and electron-ion correlation functions; the result is given in terms of the electron distribution function, which is left arbitrary. In Sec. III an expression for the conductivity is presented, where the ion-ion correlations appear explicitly; for a moderate field (moderate in the sense that the resulting directed component of electron velocity is small compared with the electron thermal velocity) we make a detailed comparison between some conductivity results of Ref. 5 and those given here. In Sec. IV we discuss the restrictions imposed by our analysis on the field intensity.

Finally, we point out some important assumptions that will be used in the analysis: (1) The driving frequency is large compared with the effective collision frequency; and (2) the field is uniform (wavelength large compared with both the electron Debye length and the excursion length of the electrons in the field). 


\section{ION-ION CORRELATIONS}

We consider an infinite, homogeneous plasma composed of electrons and one species of ions, inside which there is a spatially uniform, oscillating electric field. From the Liouville equation, the usual BBGKY hierarchy ${ }^{6}$ is derived. The equations for the one-particle distribution function of the $\alpha$ species, $f^{\alpha}$, and the correlation function between two particles of species $\alpha$ and $\beta, g^{\alpha \beta}$, read

$$
\begin{aligned}
& \frac{\partial f_{1}^{\alpha}}{\partial t}+\frac{q_{\alpha}}{m_{\alpha}} \mathbf{E} \sin \omega t \cdot \frac{\partial f_{1}^{\alpha}}{\partial \mathrm{v}_{1}} \\
& \quad=\frac{q_{\alpha}}{m_{\alpha}} \frac{\partial}{\partial \mathrm{v}_{1}} \cdot \int d \mathbf{r}_{2} d \mathbf{v}_{2} \frac{\partial \phi_{12}}{\partial \mathbf{r}_{1}} \sum_{\beta} q_{\beta} n_{\beta} g_{12}^{\alpha \beta}, \\
& {\left[\frac{\partial}{\partial t}+\left(\mathbf{v}_{1}-\mathrm{v}_{2}\right) \cdot \frac{\partial}{\partial \mathbf{r}_{12}}\right.} \\
& \left.+\mathbf{E} \sin \omega t \cdot\left(\frac{q_{\alpha}}{m_{\alpha}} \frac{\partial}{\partial \mathbf{v}_{1}}+\frac{q_{\beta}}{m_{\beta}} \frac{\partial}{\partial \mathbf{v}_{2}}\right)\right] g_{12}^{\alpha \beta} \\
& =q_{\alpha} q_{\beta} \frac{\partial \phi_{12}}{\partial \mathbf{r}_{12}} \cdot\left(\frac{1}{m_{\alpha}} \frac{\partial}{\partial \mathrm{v}_{1}}-\frac{1}{m_{\beta}} \frac{\partial}{\partial \mathrm{v}_{2}}\right) f_{1}^{\alpha} f_{2}^{\beta} \\
& \quad+\frac{q_{\alpha}}{m_{\alpha}} \frac{\partial f_{1}^{\alpha}}{\partial \mathrm{v}_{1}} \cdot \frac{\partial}{\partial \mathbf{r}_{12}} \int d \mathbf{r}_{3} \phi_{13} \int d \mathbf{v}_{3} \sum_{\gamma} q_{\gamma} n_{\gamma} g_{23}^{\beta \gamma} \\
& +\frac{q_{\beta}}{m_{\beta}} \frac{\partial f_{2}^{\beta}}{\partial \mathrm{v}_{2}} \cdot \frac{\partial}{\partial \mathrm{r}_{12}} \int d \mathbf{r}_{3} \phi_{23} \int d \mathbf{v}_{3} \sum_{\gamma} q_{\gamma} n_{\gamma} g_{13}^{\alpha \gamma},
\end{aligned}
$$

where $\phi_{12}=r_{12}^{-1}, \mathbf{r}_{12}=\mathbf{r}_{1}-\mathbf{r}_{2}, \mathbf{E}$ sin $\omega t$ is the field prevailing inside the plasma and, because of the homogeneity of the plasma, $r_{1}$ and $\mathbf{r}_{2}$ only appear in (2) through their difference; the subscripts are particle indices and $\alpha, \beta, \gamma$ stand for $e$, electrons, or $i$, ions. Notice that in (2) two terms are missing from the exact correlation equation: One is $\partial \phi_{12} /$ $\partial \mathbf{r}_{12} \cdot\left(m_{\alpha}^{-1} \partial / \partial \mathbf{v}_{1}-m_{\beta}^{-1} \partial / \partial \mathbf{v}_{2}\right) q_{\alpha} q_{\beta} g_{12}^{\alpha \beta}$, the other involves the three-particle correlation function $h^{\alpha \beta \gamma}$. Both terms are usually neglected in plasma kinetic theory ${ }^{6}$; we shall now assume that here they are likewise negligible, and delay a discussion of the restrictions that such assumption may impose on the field intensity until Sec. IV.

Before studying Eq. (2) we consider (1) briefly. We assume that $\nu / \omega<O(1)$, where $\nu$ is the collision frequency ${ }^{7}$; there are then two widely separated time scales, with characteristic times $\omega^{-1}$ and $\nu^{-1}$. Expanding $f_{1}^{\alpha}$ in powers of $\nu / \omega$,

$$
f_{1}^{\alpha}=f_{1}^{\alpha 0}+(\nu / \omega) f_{1}^{\alpha 1}+\cdots,
$$

we have to lowest order

$$
\frac{\partial f_{1}^{\alpha 0}}{\partial t}+\frac{q_{\alpha}}{m_{\alpha}} \mathbf{E} \sin \omega t \cdot \frac{\partial f_{1}^{\alpha 0}}{\partial \mathbf{v}_{1}}=0,
$$

that is, in this frequency range and to lowest order, the driving field is balanced by the inertial force. The solution to (4) is an arbitrary function of $\mathfrak{u}_{1}^{\alpha} \equiv \mathbf{v}_{1}+\omega \varepsilon_{\alpha} \cos \omega t$, where $\varepsilon_{\alpha} \equiv q_{\alpha} \mathbf{E} \cdot\left(m_{\alpha} \omega^{2}\right)^{-1}$ is the oscillation excursion of particle $\alpha ; f_{1}^{\alpha 0}$, which is normalized to unity, describes a purely reactive response to the field. A discussion of the character of the function $f_{1}^{\alpha 0}\left(\mathbf{u}_{1}^{\alpha}\right)$ is included in the next section; in solving for the correlations below we let $f_{1}^{\alpha 0}$ be an even, arbitrary function of $\mathfrak{u}_{1}^{\alpha}$.

With this result for $f_{1}^{\alpha 0}$, we can now proceed to a determination of the ion-ion correlations. Equation (2) represents three coupled equations for $g^{e \theta}, g^{e i}$, and $g^{i i}$. It will be seen below that in the limit $m_{i} \rightarrow \infty$ (to be taken later in the section) the $g^{e i}, g^{i i}$ equations become uncoupled from the equation for $g^{e \theta}$. Thus, to solve for $g^{i i}$ we need only consider the $g^{e i}, g^{i i}$ equations.

First consider Eq. (2) with $\alpha=e, \beta=i$. To lowest order in $\nu / \omega$ we solve for $g^{e i}$ by approximating $f_{1}^{e}, f_{2}^{i}$ by $f_{1}^{i 0}, f_{2}^{i 0}$ on the right-hand side of (2). Then introducing the transformation

$$
\begin{gathered}
t=t, \quad \mathfrak{u}_{1}^{e}=\mathrm{v}_{1}+\omega \varepsilon_{e} \cos \omega t, \\
\mathfrak{u}_{2}^{i}=\mathrm{v}_{2}+\omega \varepsilon_{i} \cos \omega t, \\
\mathbf{0}_{12}=\mathbf{r}_{12}+\varepsilon_{e i} \sin \omega t \quad\left(\boldsymbol{\varepsilon}_{e i}=\varepsilon_{e}-\varepsilon_{i}\right)
\end{gathered}
$$

(that is, measuring the coordinates of each particle in its own oscillating frame) the equation for $g_{12}^{e i}$ becomes

$$
\begin{aligned}
& {\left[\frac{\partial}{\partial t}+\left(\mathbf{u}_{1}^{e}-\mathfrak{u}_{2}^{i}\right) \cdot \frac{\partial}{\partial \varrho_{12}}\right] g_{12}^{e,}} \\
& =q_{e} q_{i} \frac{\partial \phi_{12}}{\partial \boldsymbol{\vartheta}_{12}} \cdot\left(\frac{1}{m_{e}} \frac{\partial}{\partial \mathbf{u}_{1}^{e}}-\frac{1}{m_{i}} \frac{\partial}{\partial \mathbf{u}_{2}^{i}}\right) f_{1}^{e 0} f_{2}^{2 n} \\
& +\frac{q_{e}^{2} n_{e}}{m_{e}} \frac{\partial f_{1}^{e 0}}{\partial \mathfrak{u}_{1}^{e}} \cdot \frac{\partial}{\partial \boldsymbol{\varrho}_{12}} \int d r_{3} \phi_{12}\left(\int g_{23}^{i e} d u_{3}^{e}-g_{23}^{i i} d \mathfrak{u}_{3}^{i}\right) \\
& -\frac{q_{e} q_{i} n_{e}}{m_{i}} \frac{\partial f_{2}^{i 0}}{\partial \mathbf{u}_{2}^{i}} \cdot \frac{\partial}{\partial \varrho_{12}} \int d \mathbf{r}_{3} \phi_{23} \\
& \cdot\left(\int g_{13}^{e e} d \mathbf{u}_{3}^{e}-\int g_{13}^{e i} d \mathbf{u}_{3}^{i}\right) \text {, }
\end{aligned}
$$

where the neutrality condition, $q_{i} n_{i}=-q_{\theta} n_{e}$, has been used. Integrating over $u_{2}^{i}$ we get

$$
\begin{aligned}
\left(\frac{\partial}{\partial t}+\right. & \left.\mathfrak{u}_{1}^{e} \cdot \frac{\partial}{\partial \varrho_{12}}\right) G_{12}^{e i} \\
= & \frac{q_{e} q_{i}}{m_{e}} \frac{\partial \phi_{12}}{\partial \varrho_{12}} \cdot \frac{\partial f_{1}^{e 0}}{\partial \mathfrak{u}_{1}^{e}}+\frac{q_{e}^{2} n_{e}}{m_{e}} \frac{\partial f_{1}^{e 0}}{\partial \mathfrak{u}_{1}^{e}} \cdot \frac{\partial}{\partial \boldsymbol{\varrho}_{12}} \int d \mathbf{r}_{3} \phi_{13} \\
& \cdot\left(\int G_{32}^{e i} d \mathfrak{u}_{3}^{e}-\int g_{32}^{i i} d \mathfrak{u}_{2}^{i} d \mathfrak{u}_{3}^{i}\right)-\frac{\partial}{\partial \varrho_{12}} \cdot \int \mathfrak{u}_{2}^{i} g_{12}^{e i} d \mathfrak{u}_{2}^{i},
\end{aligned}
$$


where $G^{o i}=\int g^{e i} d \mathbf{u}^{i}$; use has been made of the physical requirements $g_{p s}^{\alpha \beta}=g_{s p}^{\beta \alpha}$ and $f_{s}^{\alpha}\left(u_{s}^{\alpha} \rightarrow \pm \infty\right)$ $\rightarrow 0$. Equation (6) is much simpler than (5), except for the presence of the last term of (6); this term, however, is expected to vanish in the limit $m_{i} \rightarrow \infty$. Notice also that $g^{e e}$ has disappeared from Eq. (6).

Let us now define the Fourier transform of any function of $\mathrm{r}_{12}, A\left(\mathrm{r}_{12}\right)$ :

$$
\widetilde{A} \equiv \int d \mathbf{r}_{12} \exp \left(-i \mathbf{k} \cdot \mathbf{r}_{12}\right) A\left(\mathbf{r}_{12}\right) .
$$

Let us also define

$$
\begin{aligned}
& \tilde{\widetilde{A}} \equiv \exp \left(-i \mathbf{k} \cdot \varepsilon_{e i} \sin \omega t\right) \tilde{A} \\
& =\int d \mathbf{0}_{12} \exp \left(-i \mathbf{k} \cdot \mathbf{0}_{12}\right) A\left(\mathbf{0}_{12}\right),
\end{aligned}
$$

which is the Fourier transform of $A$ considered as a function of $0_{12}$. Then, Fourier analyzing (6) with respect to $\mathbf{0}_{12}$, we get

$$
\begin{aligned}
\left(\frac{\partial}{\partial t}+i \mathbf{k} \cdot \mathbf{u}_{1}^{e}\right) \widetilde{G}_{12}^{e i} \\
=\frac{q_{e} q_{i}}{m_{e}} i \mathbf{k} \cdot \frac{\partial f_{1}^{e 0}}{\partial \mathbf{u}_{1}^{e}} \frac{4 \pi}{k^{2}} \exp \left(-i \mathbf{k} \cdot \varepsilon_{e i} \sin \omega t\right) \\
\quad+\frac{q_{e}^{2} n_{e}}{m_{e}} i \mathbf{k} \cdot \frac{\partial f_{1}^{e 0}}{\partial \mathbf{u}_{1}^{e}} \frac{4 \pi}{k^{2}} \\
\quad \cdot\left[\int \widetilde{\widetilde{G}}_{32}^{e i} d \mathbf{u}_{3}^{e}-\exp \left(-i \mathbf{k} \cdot \varepsilon_{e i} \sin \omega t\right) \int \tilde{g}_{32}^{i i} d \mathbf{u}_{2}^{i} d \mathbf{u}_{3}^{i}\right] \\
\quad-i \mathbf{k} \cdot \int \mathbf{u}_{2}^{e}{\widetilde{g_{12}^{e i}}}_{12}^{e i} d \mathbf{u}_{2}^{i},
\end{aligned}
$$

where $\tilde{\phi}=4 \pi k^{-2}$ has been used. We solve for $\widetilde{G}^{e i}$ in (7) under the assumption of vanishing perturbations in the remote past and then integrate over $\mathfrak{u}_{1}^{o}$ to obtain

$$
\begin{gathered}
\widetilde{\widetilde{Q}}^{\epsilon i}(t)=\frac{\omega_{p}^{2}}{k^{2}} \int d \mathbf{u}_{1}^{e} i \mathbf{k} \cdot \frac{\partial f_{1}^{e 0}}{\partial \mathbf{u}_{1}^{e}} \int_{-\infty}^{t} d \tau \exp \left[-i \mathbf{k} \cdot \mathbf{u}_{1}^{e}(t-\tau)\right] \\
\cdot\left[\widetilde{\widetilde{Q}}^{e i}(\tau)-\exp \left(-i \mathbf{k} \cdot \varepsilon_{e i} \sin \omega \tau\right)\right. \\
\left.\quad\left(\frac{1}{n_{i}}+\int \tilde{g}_{32}^{i i}(\tau) d \mathbf{u}_{2}^{i} d \mathbf{u}_{3}^{i}\right)\right]-\int_{-\infty}^{t} d \tau \int \widetilde{\tilde{g}}_{12}^{e i}(\tau) \\
\cdot \exp \left[-i \mathbf{k} \cdot \mathbf{u}_{1}^{e}(t-\tau)\right] i \mathbf{k} \cdot \mathbf{u}_{2}^{i} d \mathbf{u}_{2}^{i} d \mathbf{u}_{1}^{e},
\end{gathered}
$$

where $\widetilde{Q}^{e i}=\int \widetilde{G}^{i i} d \mathbf{u}^{0}$ and $\omega_{p}^{2}=4 \pi q_{e}^{2} n_{e} / m_{e}$.

Let us make the ansatz that for large $m_{i}$,

$$
\begin{gathered}
f^{i 0}=f_{M}\left(\mathrm{u}^{i}\right)+o(1), \quad g^{e i}=f^{i 0} G^{e i}+o(1), \\
g_{a p}^{i i}=\sum_{l} g_{s p l l}^{i i} \exp (-i l \omega \tau)+o(1), \\
g_{s p(0}^{i i}=f_{s}^{i 0} f_{p}^{i 0} Q^{i i}\left(\mathrm{r}_{s p}\right)+o(1),
\end{gathered}
$$

where $f_{M}$ is a Maxwellian distribution and the symbol o(1) represents a small quantity that goes to zero as $m_{i} \rightarrow \infty$. The last term in (8) now vanishes and writing

$$
\exp \left[-i \mathbf{k} \cdot \varepsilon_{e} \sin \omega \tau\right]=\sum_{l} J_{l}\left(\mathbf{k} \cdot \varepsilon_{e}\right) \exp (-i l \omega \tau)
$$

we obtain for $\widetilde{\widetilde{Q}}^{i i}$, in the limit $m_{i} \rightarrow \infty$,

$$
\begin{gathered}
{\widetilde{Q^{e}}}^{i i}=\sum_{l}{\widetilde{Q^{\prime}}}_{(i}^{i i} \exp (-i l \omega \tau), \\
\widetilde{Q}_{(l}^{e i}=\frac{J_{l \chi} \chi_{-l}}{n_{i} D_{-l}}\left(1+n_{1} \widetilde{Q}^{i i}\right) \\
+\frac{\chi_{-l}}{D_{-l}} \sum_{l^{\prime} \neq 0} J_{l-l}, \int \tilde{g}_{\left(l^{\prime}\right.}^{i i} d \mathbf{u}^{i} d \mathbf{u}^{i},
\end{gathered}
$$

where

$D_{-\imath} \equiv 1+\chi_{-\imath} \equiv 1$

$$
+\frac{\omega_{p}^{2}}{k^{2}} \int \frac{\mathbf{k} \cdot \partial f_{1}^{\rho 0} / \partial \mathbf{u}_{1}^{e}}{i \omega-\mathbf{k} \cdot \mathbf{u}_{1}^{\sigma}+i \delta} d \mathbf{u}_{1}^{o}, \quad\left(\delta \rightarrow 0^{+}\right) .
$$

Let us now consider Eq. (2) with $\alpha=\beta=i$. Introducing the transformation

$$
t=t, \quad \mathbf{u}_{1}^{i}=\mathrm{v}_{1}+\omega \varepsilon_{i} \cos \omega t, \quad \mathbf{u}_{2}^{i}=\mathrm{v}_{2}+\omega \varepsilon_{i} \cos \omega t,
$$

and Fourier analyzing with respect to $\mathbf{r}_{12}$ we obtain

$$
\begin{aligned}
{\left[\frac{\partial}{\partial t}+\right.} & \left.i \mathbf{k} \cdot\left(\mathbf{u}_{1}^{i}-\mathbf{u}_{2}^{i}\right)\right] \tilde{g}_{12}^{i i} \\
= & \frac{q_{i}^{2}}{m_{i}} i \mathbf{k} \frac{4 \pi}{k^{2}} \cdot\left(\frac{\partial}{\partial \mathbf{u}_{1}^{i}}-\frac{\partial}{\partial \mathbf{u}_{2}^{i}}\right) f_{1}^{i 0} f_{2}^{i 0}+\frac{q_{i} q_{e} n_{e}}{m_{i}} i \mathbf{k} \cdot \frac{\partial f_{1}^{i 0}}{\partial \mathbf{u}_{1}^{i}} \frac{4 \pi}{k^{2}} \\
& \cdot\left[\exp \left(i \mathbf{k} \cdot \varepsilon_{e i} \sin \omega t\right) \int \tilde{g}_{32}^{e i} d \mathbf{u}_{3}^{e}-\int \tilde{g}_{32}^{i i} d \mathbf{u}_{i}^{3}\right] \\
& -\frac{q_{i} q_{e} n_{e}}{m_{i}} i \mathbf{k} \cdot \frac{\partial f_{2}^{i 0}}{\partial \mathbf{u}_{2}^{i}} \frac{4 \pi}{k^{2}} \\
& \cdot\left[\exp \left(-i \mathbf{k} \cdot \varepsilon_{e i} \sin \omega t\right) \int \tilde{g}_{13}^{i e} d \mathbf{u}_{3}^{o}-\int \tilde{g}_{13}^{i i} d \mathbf{u}_{3}^{i}\right]
\end{aligned}
$$

Now using the ansatz in (9), we obtain for $g_{12(1)}^{i i}$ $l \neq 0$

$$
\begin{aligned}
& {\left[-l \omega+\mathbf{k} \cdot\left(\mathfrak{u}_{1}^{i}-\mathfrak{u}_{2}^{i}\right)\right] \hat{g}_{12 \ell}^{i i}=\frac{q_{0} q_{i} n_{0}}{\kappa T_{i}} \frac{4 \pi}{k^{2}} \mathbf{k}} \\
& \cdot\left[\mathfrak{u}_{1}^{i} f_{M}\left(\mathbf{u}_{1}^{i}\right) \int \tilde{g}_{32 \ell l}^{i i} d \mathbf{u}_{3}^{i}-\mathbf{u}_{2}^{i} f_{M}\left(\mathbf{u}_{2}^{i}\right) \int \tilde{g}_{\mathbf{3} 3 l}^{i i} d \mathbf{u}_{3}^{i}\right] \\
& -\frac{q_{\epsilon} q_{i} n_{e}}{m_{i}} \frac{4 \pi}{k^{2}} \mathbf{k} f_{M}\left(\mathfrak{u}_{1}^{i}\right) f_{M}\left(\mathfrak{u}_{2}^{i}\right) \\
& \cdot\left[\mathbf{u}_{1}^{i} \sum_{l^{\prime}} J_{l^{\prime}} \widetilde{\widetilde{Q}}_{\left(l+l^{\prime}\right.}^{i}-\mathbf{u}_{2}^{i} \sum_{l^{\prime}} J_{l^{\prime}} \widetilde{\widetilde{Q}}_{\left(l-l, l^{\prime}\right.}^{i},(-\mathbf{k})\right] \\
& +o(1), \quad l \neq 0,
\end{aligned}
$$


where use has been made of the identity $\widetilde{\widetilde{Q}}^{i e}(\mathrm{k})=$ $\widetilde{\widetilde{Q}}^{e i}(-\mathbf{k})$. In the limit $m_{i} \rightarrow \infty$ we have $f_{M}\left(\mathbf{u}^{i}\right) \rightarrow$ $\delta\left(\mathbf{u}^{i}\right)$ and since $\mathbf{u} \delta(\mathbf{u}) \equiv 0$, we get $g_{(l}^{i}=0$ for $l \neq 0$. Thus, the last term in (10b) vanishes. For $g_{(0}^{2 i}$ we get from (11)

$$
\begin{aligned}
\mathbf{k} \cdot\left(\mathbf{u}_{1}^{i}-\mathbf{u}_{2}^{i}\right) \tilde{g}_{12(0}^{i i} \\
=-\frac{q_{i}^{2}}{\kappa T_{i}} \frac{4 \pi}{k^{2}} \mathbf{k} \cdot\left(\mathbf{u}_{1}^{i}-\mathfrak{u}_{2}^{i}\right) f_{M}\left(\mathfrak{u}_{1}^{i}\right) f_{M}\left(\mathbf{u}_{2}^{i}\right) \\
\quad+\frac{q_{e} q_{2} n_{c}}{\kappa T_{i}} \frac{4 \pi}{k^{2}} \mathbf{k} \cdot\left(\mathbf{u}_{1}^{i}-\mathbf{u}_{2}^{i}\right) \tilde{g}_{12(0}^{i i} \\
\quad-\frac{q_{e} q_{i} n_{0}}{\kappa T_{i}} \frac{4 \pi}{k^{2}} f_{M}\left(\mathbf{u}_{i}^{i}\right) f_{M}\left(\mathbf{u}_{2}^{i}\right) \\
\quad \cdot \mathbf{k} \cdot\left[\mathbf{u}_{1}^{i} \sum_{l} J_{l} \widetilde{Q}_{(l}^{e i}-\mathbf{u}_{2}^{i} \sum_{l} J_{l} \widetilde{Q}_{(-l}^{e i}(-\mathbf{k})\right]+o(\mathbf{1}) .
\end{aligned}
$$

Introducing $\tilde{g}_{<0}^{i i}$ and $\widetilde{\widetilde{Q}}_{(l)}^{e i}$, as given in (9) and (10), into (12), dividing by $f_{M}\left(\mathbf{u}_{1}^{i}\right) f_{M}\left(\mathfrak{u}_{2}^{i}\right)$ and taking the limit $m_{i} \rightarrow \infty$, we obtain

$$
\begin{aligned}
\mathbf{k} \cdot\left(\mathrm{u}_{1}^{i}-\mathfrak{u}_{2}^{i}\right)\left[\widetilde{Q}^{i i}+\frac{q_{i}^{2}}{\kappa T_{i}} \frac{4 \pi}{k^{2}}-\frac{q_{e} q_{i} n_{e}}{\kappa T_{i}} \frac{4 \pi}{k^{2}} \widetilde{Q}^{i i}\right. \\
\left.+\frac{q_{e} q_{2} n_{e}}{\kappa T_{i}} \frac{4 \pi}{k^{2}} \sum_{l} \frac{J_{l}^{2} \chi_{-1}}{n_{i} D_{-l}}\left(1+n_{\imath} \widetilde{Q}^{i i}\right)\right]=0
\end{aligned}
$$

where the symmetry of $\widetilde{Q}^{i i}, \widetilde{Q}^{i i}(-\mathbf{k})=\widetilde{Q}^{i i}(\mathbf{k})$, has been used. From (13)

$$
\widetilde{Q}^{i i}=-\frac{1}{n_{l}} \frac{k_{i}^{2} \sum_{l} J_{l}^{2} / D_{-l}+k_{i}^{2} \sum_{l} J_{l}^{2} / D_{-l}}{},
$$

which is the desired result for the ion-ion correlations; we have defined $k_{\alpha}^{2} \equiv 4 \pi q_{\alpha}^{2} n_{\alpha} /{ }^{\prime} T_{\alpha}$.

Notice that $\widetilde{Q}^{i i}$ is a function of both the frequency and the intensity of the field. As $E \rightarrow 0, \widetilde{Q}^{i i} \rightarrow$ $-n_{i}^{-1} k_{i}^{2}\left(k_{i}^{2}+k^{2} D_{0}\right)^{-1}$ and since $f^{e 0}$ should then be Maxwellian we recover the thermal correlations

$$
\widetilde{Q}^{i i}=-\frac{1}{n_{i}} \frac{k_{i}^{2}}{k_{i}^{2}+k_{e}^{2}+k^{2}}+O\left(E^{2}\right)
$$

for small $E$ the corrections are $O\left(E^{2}\right)$ so that the effect is essentially nonlinear. The ion-ion correlation function is obtained from $g^{i i} \equiv f_{M I}^{i} f_{M}^{i} Q^{i i}$ and (14). Using (14) we can also obtain $g^{e i}$ from $g^{e i} \equiv$ $f_{M}^{i} G^{e i}$, Eq. (10) and Eq. (7), which may now be easily solved. Finally, we can go back and observe that our ansatz $g^{e i}=f_{M}^{i} G^{e i}$ satisfies (5) in the limit $m_{i} \rightarrow \infty$ [notice simply Eq. (6) and the identity $\mathbf{u}^{i} \delta\left(\mathrm{u}^{i}\right) \equiv 0$ ]. Thus, by assuming the ansatz in (9) we have obtained a consistent solution of the equations for $g^{e i}$ and $g^{i i}$ as $m_{i} \rightarrow \infty$. We remark that this ansatz has placed no restriction on $g^{e e}$.

\section{THE NONLINEAR HIGH. FREQUENCY PLASMA CONDUCTIVITY}

The influence of the above effect on the electric conductivity will now be studied. To determine the conductivity we must find the current-density response to the field,

$$
\mathbf{j}=\sum_{\alpha} \mathbf{j}^{\alpha} \equiv \sum_{\alpha} n_{\alpha} q_{\alpha} \int f^{\alpha} \mathrm{v} d \mathbf{v} .
$$

Since $m_{i} \rightarrow$ oo, only the electron distribution function has to be considered; using the expansion in (3) with $\alpha=e$, we get

$$
\begin{aligned}
\mathrm{j}=\mathrm{j}^{e 0}+\frac{\nu}{\omega} \mathrm{j}^{e 1}+\cdots \equiv n_{e} q_{\varepsilon} \\
\quad \cdot \int f^{\theta 0} \mathrm{v} d \mathrm{v}+\frac{\nu}{\omega} n_{e} q_{e} \int f^{e 1} \mathrm{v} d \mathrm{v}+\cdots .
\end{aligned}
$$

For $f^{e 0}\left(\mathbf{u}^{e}\right)$ even in $\mathbf{u}^{e}, \mathbf{j}^{e 0}$ is purely reactive,

$$
\mathrm{j}^{r 0}=-n_{e} q_{\theta} \omega \varepsilon_{e} \cos \omega t
$$

and to obtain a resistive component of $\mathbf{j}$ (of most interest because it produces heating of the plasma), we must find $\mathbf{j}^{\mathbf{1}}$.

It is now interesting to notice the following point: The resistive current, to be found below, heats the plasma slowly (in the $\nu^{-1}$ scale). Unless this heating is taken into account by allowing a slow temperature variation in $f^{\circ}$, the expansion in (3) will ultimately break down due to secular behavior ${ }^{8}$; actually, there exists not only heating but a general broadening of $f^{e 0}$, which will not be Maxwellian. The introduction of the slow-time variable means that $f^{60}$ only satisfies (4) in the $\omega^{-1}$, fast scale; the whole analysis of Sec. II only yields the fast-time dependence in the equations, but the results are still valid because the slow-time variable would appear merely as a parameter. The determination of $f^{e 0}\left(\mathfrak{u}^{e}\right)$ involves the study of the slow-time evolution of the system, and, in particular, requires solving the equation for $g^{e e}$; this will not be done here. Thus, our result (14), gives $\widetilde{Q}^{i i}$ as a functional of $f^{e 0}$.

Fortunately, $\mathrm{j}^{\mathrm{e} 1}$ may be obtained (as a functional of $f^{e 0}$ again) without studying the slow-time evolution of the plasma. This is so because, as assumed in Sec. II, $f^{e 0}$ has to be even in $\mathrm{u}^{e}$ : for times comparable to $\nu^{-1}$, there is no preferential direction along an axis aligned with the field, since $\mathbf{E}$ sin $\omega t$ changes sign every half-period. Then, consider Eq. (1) with $\alpha=e$, multiply by $n_{e} q_{e} \mathbf{v}_{1}$ and integrate over $\mathrm{v}_{1}$; the result is

$$
\frac{\partial \mathrm{j}^{\delta}}{\partial t}-\frac{q_{e}^{2} n_{e}}{m_{e}} \mathbf{E} \sin \omega t=\frac{q_{e}^{3} n_{e}^{2}}{m_{e}} \int d \mathbf{r}_{2} \frac{\partial \phi_{12}}{\partial \mathbf{r}_{12}} \int g_{12}^{e i} d \mathbf{v}_{1} d \mathrm{v}_{2} \text {. }
$$


Since $f^{o 0}$ is even in $\mathfrak{u}^{e}$, (16) shows that the equation

$$
\frac{\partial \mathrm{j}^{e 0}}{\partial t}=\frac{q_{e}^{2} n_{e}}{m_{e}} \mathrm{E} \sin \omega t
$$

is satisfied not only in the fast scale but identically. Thus, after introducing (15) into (17), we obtain $\frac{\nu}{\omega} \frac{\partial \mathbf{j}^{e 1}}{\partial t}=-\frac{q_{e}^{3} n_{e}^{2}}{m_{e}}(2 \pi)^{-\mathbf{3}} \int i \mathbf{k} \tilde{\boldsymbol{\phi}} d \mathbf{k} \int \tilde{g}_{12}^{e i} d \mathbf{u}_{1}^{e} d \mathbf{u}_{2}^{i}$,

where $\tilde{\phi}$ and $\tilde{g}^{e i}$ were defined in Sec. II. Using the value of $\widetilde{Q}^{e i}$ given by Eq. (10) and integrating over $t$, (18) becomes

$$
\begin{aligned}
\frac{\nu}{\omega} \mathrm{j}^{\mathrm{j}^{1}}=-\frac{q_{e}^{2} n_{e} q_{i}}{2 \pi^{2} m_{e} \omega} \int \frac{\mathbf{k} d \mathbf{k}}{k^{2}} \sum_{l} \exp (i l \omega t) \\
\cdot \sum_{l^{\prime}} \frac{J_{l^{\prime}} J_{l+l^{\prime}}}{l D_{-l^{\prime}}}\left(1+n_{i} \widetilde{Q}^{i i}\right),
\end{aligned}
$$

$\widetilde{Q}^{i i}$ being given by (14). The result from Ref. 5 may be recovered by writing $\widetilde{Q}^{i i}=0$ and $f^{e 0}=$ $f_{M}\left(\mathfrak{u}^{e}\right)$ in the above equation.

The conductivity can be obtained from (15), (16), and (19) through the usual definition, $j=\sigma \mathrm{E}$ sin $\omega t$; actually, a series of conductivity coefficients should be defined, because all harmonics of the fundamental frequency appear in $\mathbf{j}$. Writing

$$
\mathbf{j}=\mathrm{E}\left(\sum_{l=1}^{\infty} \sigma_{l} \sin l \omega t+\sum_{l=1}^{\infty} \sigma_{l}^{\prime} \cos l \omega t\right),
$$

$\sigma_{\iota}$ and $\sigma_{l}^{\prime}$ may be immediately obtained from (16) and (19).

The actual computation of $\sigma_{l}$ and $\sigma_{l}^{\prime}$ cannot be carried out because $f^{e 0}$ is unknown. However, the most interesting aspect of our result for $Q^{i i}$ is the appearance in (14) of all resonant factors, $\left(D_{-l}\right)^{-1}$; to determine the importance of this we now compare the result of Ref. 5 with ours for a simple case, that of a moderate field $\left(\left|\omega \varepsilon_{e}\right| \ll v_{e}\right.$, $v_{\theta}$ being the electron thermal velocity).

To make the comparison simpler we only consider $\sigma_{1}$, corresponding to the component of $j$ that heats the plasma. From (19) we have, after using (14),

$$
\begin{aligned}
& \mathrm{E} \sigma_{1}=\frac{\omega_{p}^{2} q_{i}}{4 \pi^{3} \omega} \int \frac{d \mathrm{kk}}{k^{2}} \sum_{l} \frac{J_{l} J_{l+1}}{\left|D_{l}\right|^{2}} \\
& \text { - Im } D_{l} \frac{k^{2}}{h^{2}+k_{i}^{2} \sum_{l^{\prime}} J_{l^{\prime}}^{\overline{2}} / D_{-l^{\prime}}} ;
\end{aligned}
$$

the corresponding result from Ref. 5 may be obtained by dropping the last fraction in (20). Retaining up to the third power of $E$, the integral in (20) becomes

$$
\int \frac{d \mathbf{k} k}{k^{2}} \frac{\mathbf{k} \cdot \varepsilon_{e}}{2} A(\mathbf{k})
$$

$$
\begin{aligned}
& A(\mathrm{k}) \equiv\left[\frac{\operatorname{Im} D_{1}}{\left|D_{1}\right|^{2}}-\frac{\left(\mathbf{k} \cdot \varepsilon_{e}\right)^{2}}{8}\left(\frac{2 \operatorname{Im} D_{1}}{\left|D_{1}\right|^{2}}-\frac{\operatorname{Im} D_{2}}{\left|D_{2}\right|^{2}}\right)\right] \\
& \cdot k^{2}\left[k^{2}+k_{i}^{2}\left(\frac{1-\left(\mathbf{k} \cdot \varepsilon_{e}\right)^{2} / 2}{D_{0}}+\frac{\left(\mathbf{k} \cdot \varepsilon_{e}\right)^{2}}{2} \frac{\operatorname{Re} D_{1}}{\left|D_{1}\right|^{2}}\right)\right]^{-1}
\end{aligned}
$$

the prefixes Re and Im stand for real and imaginary parts. At this point we remark that the small argument expansion for the Bessel functions of (20), used to obtain Eq. (21b), is valid under the moderate field assumption even if $\left|\mathbf{k} \cdot \varepsilon_{e}\right|$ is not small. This may be proved by following a procedure used in Refs. 4 and 5: when $\left|\mathrm{k} \cdot \varepsilon_{\theta}\right| \geq O$ (1), we have $\omega / l v_{0} \ll 1$ from our condition $\left|\omega \varepsilon_{o}\right| \ll v_{e}$; then one solves (8) by expanding $\widetilde{Q}^{i i}(\tau)$ and exp $\left(-i k \cdot \varepsilon_{e} \sin \omega \tau\right)$ inside the time integral in a Taylor series in $\omega / k v_{e}$, and solving iteratively for $\widetilde{Q}^{e i}(t)$. The result for $\sigma_{1}$ agrees with that obtained from (21 b) by expanding $D_{1}\left(\omega / k v_{e}\right), D_{2}\left(2 \omega / k v_{o}\right)$ for small $\omega / k v_{c}$.

To make our comparison simpler we further expand the second bracket in (21b), although this is not valid throughout the whole range of the $\mathrm{k}$ integration; $k^{2}$ over this bracket becomes

$$
\begin{aligned}
& \frac{k^{2}+k_{e}^{2}}{k^{2}+k_{e}^{2}+k_{i}^{2}}\left\{1-\frac{k_{i}^{2}}{k^{2}+k_{e}^{2}+k_{i}^{2}}\right. \\
& \left.\cdot\left[-\frac{\left(\mathbf{k} \cdot \varepsilon_{e}\right)^{2}}{2}+\frac{k^{2}+k_{e}^{2}}{k^{2}} \frac{\left(\mathbf{k} \cdot \varepsilon_{e}\right)^{2}}{2} \frac{\operatorname{Re} D_{1}}{\left|D_{1}\right|^{2}}+O\left(\frac{\omega^{2} \varepsilon_{e}^{2}}{v_{e}^{2}}\right)\right]\right\} .
\end{aligned}
$$

The term $O\left(\omega^{2} \varepsilon_{e}^{2} / v_{e}^{2}\right)$ is due to the fact that $f^{e 0}=$ $f_{M}^{e}\left[1+O\left(\omega^{2} \varepsilon_{e}^{2} / v_{e}^{2}\right)\right]$ and, therefore, $D_{0}=\left(1+k_{e}^{2} / k^{2}\right)$ $\left[1+O\left(\omega^{2} \varepsilon_{c}^{2} / v_{o}^{2}\right)\right]$.

We find, finally, that the linear part of $A$ is $\left(\operatorname{Im} D_{1} /\left|D_{1}\right|^{2}\right)\left[\left(k^{2}+k_{e}^{2}\right) /\left(k^{2}+k_{e}^{2}+k_{i}^{2}\right)\right]$, while from Ref. 5 we would have $\operatorname{Im} D_{1} /\left|D_{1}\right|^{2}$; the difference is due to the use, in Ref. 5 , of zero correlations instead of the thermal value, which is the appropriate limit for a vanishingly small field. This difference, however, is not very significant, as indicated in the introduction. On the other hand, for the nonlinear part of $A$ we have

$$
\begin{aligned}
{\left[-\frac{\left(\mathrm{k} \cdot \varepsilon_{e}\right)^{2}}{8}\left(\frac{2 \operatorname{Im} D_{1}}{\left|D_{1}\right|^{2}}-\frac{\operatorname{Im} D_{2}}{\left|D_{2}\right|^{2}}\right)+\frac{\operatorname{Im} D_{1}}{\left|D_{1}\right|^{2}} O\left(\frac{\omega^{2} \varepsilon_{e}^{2}}{v_{e}^{2}}\right)\right] } \\
\quad \frac{k^{2}+k_{e}^{2}}{k^{2}+k_{e}^{2}+k_{i}^{2}}-\frac{\operatorname{Im} D_{1}}{\left|D_{1}\right|^{2}} \frac{\left(k^{2}+k_{e}^{2}\right) k_{i}^{2}}{\left(k^{2}+k_{e}^{2}+k_{i}^{2}\right)^{2}} \\
\quad \cdot\left[-\frac{\left(\mathbf{k} \cdot \varepsilon_{e}\right)^{2}}{2}+\frac{\left(\mathbf{k} \cdot \varepsilon_{e}^{2}\right.}{2} \frac{k^{2}+k_{e}^{2}}{k^{2}} \frac{\operatorname{Re} D_{1}}{\left|D_{1}\right|^{2}}+O\left(\frac{\omega^{2} \mathbf{\varepsilon}_{e}^{2}}{v_{e}^{2}}\right)\right],
\end{aligned}
$$


while Ref. 5 gives

$$
-\frac{\left(\mathbf{k} \cdot \varepsilon_{e}\right)^{2}}{8}\left(\frac{2 \operatorname{Im} D_{1}}{\left|D_{1}\right|^{2}}-\frac{\operatorname{Im} D_{2}}{\left|D_{2}\right|^{2}}\right) .
$$

Here the difference is significant; in fact, the term in (22) proportional to $\operatorname{Im} D_{1} \operatorname{Re} D_{1} /\left|D_{1}\right|^{4}$ seems to yield the dominant nonlinear contribution in the interesting case $\omega \approx \omega_{p}$. We conclude that the quantitative results given in Ref. 5 for the nonlinear high-frequency conductivity should be substantially in error, especially so if $\omega$ is near the electron plasma frequency (of most interest, for instance, in the study of heating of plasmas by lasers).

\section{DISCUSSION}

We have shown that a high-frequency electric field modifies the ion-ion correlations of a fully ionized plasma in the limit of infinite ion mass. We have derived an expression for the correlation spectrum in terms of the electron distribution function; for a vanishingly small field, our result reduces to the known thermal spectrum. We have further shown that the above effect has a substantial influence on the nonlinear high-frequency plasma conductivity.

Several simplifications have been used in our analysis; they will be now discussed. First, we assumed a uniform field with a single frequency, $\mathrm{E} \sin \omega t$. Although all harmonics of the fundamental frequency appeared in the current density and should appear, therefore, in the field itself, the monocromatic assumption is valid because for $\nu / \omega \ll 1$, we have $\left|j_{1} \nu / \omega\right| \ll\left|j_{0}\right|$. The uniformity assumption requires, as indicated in Sec. I, a wavelength large compared with both Debye length and electron excursion length. For consideration of both assumptions in the propagation of a moderately strong electromagnetic wave, see Ref. 5.

Second, we assumed that after transient effects died off, an equilibrium would be reached. Obviously, this will not be the case if the plasma is made unstable by the presence of the field. It is known that for $\omega \approx \omega_{p}$ an instability sets in for moderate fields $\left(\omega\left|\varepsilon_{\theta}\right| \ll v_{\theta}\right)^{9}$; for $\omega<\omega_{p}$, the plasma is unstable too, but the threshold is much larger $\omega\left|\varepsilon_{e}\right| / v_{e}=O(1) .^{10}$ We can briefly summarize this by stating that for $\omega \lesssim \omega_{p}$ our analysis is invalid unless the field intensity is below the appropriate threshold for instability. ${ }^{9,10}$

Third, it was assumed that $\nu(E) / \omega<O(1)$. For $E \rightarrow 0$, we have the linear result ${ }^{*}$

$$
\frac{\left.j_{1} \text { (linear) }\right)}{j_{0}} \sim \frac{\nu(E=0)}{\omega} \sim \frac{\omega_{p}}{\omega} k_{e} h \ln \Lambda,
$$

$h$ being the classical distance of closest approach and $\ln \Lambda$ the usual Coulomb logarithm; on the other hand, the nonlinear analysis of Ref. 5 shows that

$$
\frac{j_{1} \text { (nonlinear) }}{j_{1} \text { (linear) }}=O\left(\frac{\omega^{2} \varepsilon_{e}^{2}}{v_{e}^{2}}\right)
$$

for $\omega^{2} \varepsilon_{c}^{2} \ll v_{e}^{2}$. These results indicate that if $\omega^{2} \varepsilon_{s}^{2} /$ $v_{\mathrm{e}}^{2} \leq O(1)$, we have $\nu(E) / \nu(E=0)=O(1)$, and the condition $\nu(E) / \omega<O(1)$ may be written

$$
\frac{\omega}{\omega_{p}} \gg k_{e} h \sim N_{D}^{-1},
$$

$N_{D}$ being the number of particles in a Debye sphere. [Since (23) shows that the order of magnitude of the ion-electron correlations is not modified, the effect of the ion-ion correlations found in our study is not expected to alter the conclusion in (24).]

Finally, the BBGKY correlation equations were simplified in Sec. II by omitting two terms. Such omission is easily justified when $\left|g^{\alpha \beta}\right| \ll f^{\alpha} f^{\beta}$. Near thermal equilibrium, this condition is satisfied for distant collisions, which are known to dominate close collisions by a factor $O(\ln \Lambda)$; however, it is reasonable to expect that for sufficiently intense fields, such simplification of the correlation equations will break down.

It seems that the breakdown does not occur as long as $\omega^{2} \varepsilon_{e}^{2} / v_{e}^{2} \leq O(1)$ and $\left|k_{e} \varepsilon_{e}\right| \leq O(1)$. Under the first assumption, the correlations between distant particles do not change in order of magnitude, as indicated in the above discussion on the ratio $\nu(E) / \omega$, while if we have both $\omega^{2} \varepsilon_{e}^{2} / v_{e}^{2} \leq O(1)$ and $\left|k_{e} \varepsilon_{\theta}\right| \leq$ $O(1)$, the ratio of the number of close collisions to that of distant ones, does not seem to be larger than for zero field ${ }^{11}$ : In the oscillating frame of the electrons the ion now fills a relative volume of the Debye sphere that is $O\left(k_{e}^{2} h^{2}\right)$ [compared with $O\left(k_{\mathrm{c}}^{3} h^{3}\right)$ for zero field] but the probability of a close interaction for an electron crossing near the line of oscillation of the ion is only $k_{e} h \omega\left|\boldsymbol{\varepsilon}_{\varepsilon}\right| / v_{\varepsilon}$.

\section{ACKNOWLEDGMENTS}

The author is highly grateful to Dr. C. Oberman for helpful discussions. He acknowledges valuable criticism from Dr. J. Dawson and Dr. P. Kaw.

This work was performed under the auspices of the United States Atomic Energy Commission, Contract No. AT (30-1)-1238.

* Present address: Department of Mechanical Engineering, Massachusetts Institute of Technology, Cambridge, Massachusetts. 
1 In thermal equilibrium, when only interparticle and for a strong field $\nu$ depends on $E$; this is also discussed in random kinetic energies exist, the ion-ion correlations do depend on the electron charge and temperature.

2 V. P. Silin, Zh. Eksp. Teor. Fiz, 47, 2254 (1964) [Sov. Phys.-JETP 20, $1510(1965)]$

${ }_{3}$ F. Albini and S. Rand, Phys. Fluids 8, 134 (1965).

4 J. Dawson and C. Oberman, Phys. Fluids 5, 517 (1962); 6 394 (1963)

5 P. Kaw and A. Salat, Phys. Fluids 11, 2223 (1968); 12, 342 (1969).

Sec. IV.

8 This difficulty does not exist in the linear case because the heating vanishes as $E^{2}$ for $E \rightarrow 0$. The difficulty is compounded here by our assumption $\tau \bar{\gamma}_{i} \rightarrow \infty$; for many plasmas $\vec{\gamma}_{i}^{-1_{y}} \geq O(1)$ so that for the times of interest the heating is already substantial.

${ }^{9}$ D. F. DuBois and M. V. Goldman, Phys. Rev. Letters 14, $544(1965)$

10 J. R. Sanmartin, Phys. Fluids (to be published).

6 D. Montgomery and D. Tidman, Plasma Kinetic Theory (MoGraw-Hill Book Company, New York, 1963), Chaps. 4 and 6. .

11 The distant collisions are rendered less effective for

$\omega \gg \omega_{p}$. Instead of the usual value of the Coulomb logarithm,

In $\Lambda \sim$ In $\left(k_{\theta} h\right)^{-1}$, we have $1 \mathrm{n}\left(v_{e} / \omega h\right)$; see Ref. 4. However, this is not an effect that increases with the field intensity. 